\title{
Early experience with the Intrepid system for transcatheter mitral valve replacement
}

\author{
Paul Sorajja ${ }^{1}$, Vinayak Bapat ${ }^{2}$ \\ ${ }^{1}$ Abbott Northwestern Hospital, Minneapolis, MN, USA; ${ }^{2}$ Columbia University Medical Center/NY Presbyterian Hospital, New York, NY, USA \\ Correspondence to: Paul Sorajja, MD. Roger L. and Lynn C. Headrick Chair, Valve Science Center, Minneapolis Heart Institute Foundation, Abbott \\ Northwestern Hospital, Minneapolis, MN 55407, USA. Email: paul.sorajja@allina.com.
}

\begin{abstract}
Severe mitral regurgitation is common and results in a poor prognosis in patients with either symptoms or severe ventricular dilatation. While mitral regurgitation has traditionally necessitated surgical repair or replacement, new transcatheter methods, such as the IntrepidTM transcatheter mitral valve replacement, has recently emerged. The Intrepid system is a circular, self-expanding, tri-leaflet bovine pericardial prosthesis housed within a nitinol frame. In early feasibility studies, the Intrepid prosthesis has been found to be effective for relief of mitral regurgitation and associated with functional improvement in the majority of survivors.
\end{abstract}

Keywords: Transcatheter; mitral; regurgitation; surgery

Submitted Jul 10, 2018. Accepted for publication Aug 23, 2018.

doi: 10.21037/acs.2018.10.03

View this article at: http://dx.doi.org/10.21037/acs.2018.10.03

\section{Introduction}

Severe mitral regurgitation (MR) is common and results in chronic volume overload, with a poor prognosis in patients with either symptoms or severe ventricular dilatation (1-3). Traditionally, MR necessitated surgical repair or replacement, however, new transcatheter methods have recently emerged. These methods offer a relatively less invasive alternative to open surgery and may help address an unmet clinical need for MR. In this report, we describe the methodology and present outcomes with the Medtronic Intrepid ${ }^{\mathrm{TM}}$ system for transcatheter mitral valve replacement (TMVR) (4).

\section{The Intrepid ${ }^{\mathrm{TM}}$ TMVR system}

The Intrepid ${ }^{\mathrm{TM}}$ TMVR system is a self-expanding, trileaflet bovine pericardial prosthesis housed within a nitinol frame. The frame consists of two parts: a circular outer fixation frame that comes in three sizes $(43,46$, or $50 \mathrm{~mm}$ diameter) and a $27-\mathrm{mm}$ circular inner stent frame $(27 \mathrm{~mm})$ (Figure 1). Implantation and fixation for anchoring is selfcentering and symmetrical. The outer frame engages the dynamic native mitral valve anatomy with a flexible atrial brim that facilitates visualization under ultrasound during implantation. Fixation of the Intrepid ${ }^{\mathrm{TM}}$ prosthesis is achieved through oversizing and design features of the outer frame that allow the prosthesis to wedge itself in the subannular mitral space. The atrial portion of the outer frame is relatively flexible and conforms to the native annulus, while the ventricular portion is relatively stiffer. Small cleats on the outer frame also act as frictional elements to engage the native mitral leaflets. The dual frame structure allows the inner frame to remain circular throughout the cardiac cycle, without intrusion from the shape and motion of the outer frame and native annulus. There is no need for rotational orientation during device implantation. Risk of left ventricular outflow tract (LVOT) obstruction is minimized with a device profile of 17 to $18 \mathrm{~mm}$. A hydraulically actuated delivery catheter is used to deliver the Intrepid ${ }^{\mathrm{TM}}$ with a 35 French (Fr) access sheath through the apex of the left ventricle, although, a transfemoral, transseptal version of the prosthesis is currently under development.

\section{Implantation procedure}

Patients with symptomatic primary or secondary MR 

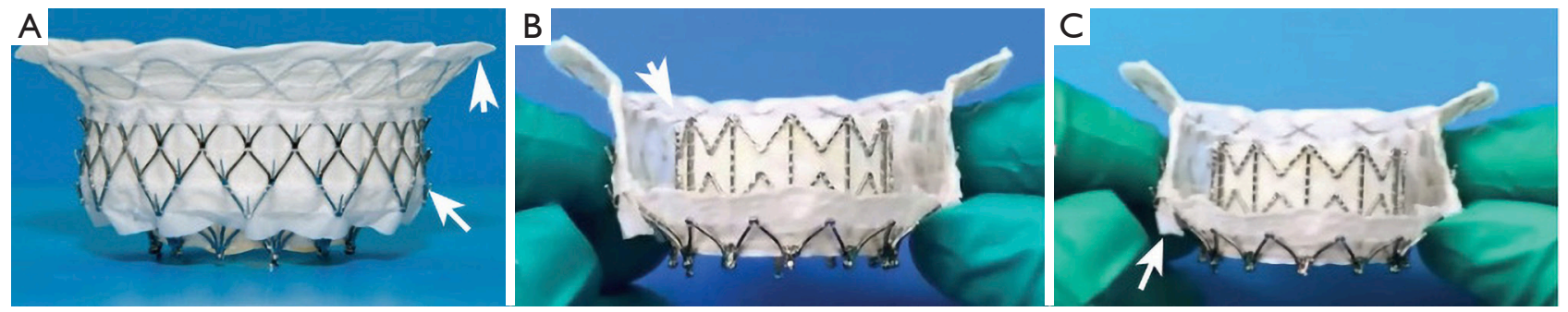

Figure 1 The Medtronic Intrepid valve. The Intrepid device is a transapical, self-expanding, symmetrical nitinol valve. (A) An atrial brim (arrowhead) is used for device placement under echocardiographic imaging, while an outer stent frame (arrow) facilitates fixation to the mitral valve annulus; (B) the inner frame houses a $27-\mathrm{mm}$ bovine pericardial valve (arrowhead); (C) the outer frame has different degrees of radial stiffness along its axial length to create a "cork-like" effect conformation that, along with small cleats, facilitates anchoring. Reproduced with permission from Bapat et al. (5).

may be considered for TMVR with the Intrepid ${ }^{\mathrm{TM}}$ system. All patients undergo contrast-enhanced, cardiac computed tomography (CT) of the mitral valve annulus to determine suitability and choice of the prosthesis size $(6,7)$. In general, a prosthesis size is chosen that will allow $10 \%$ to $30 \%$ oversizing in the mitral annular perimeter, inter-commissural diameter and septal-lateral diameter, while minimizing risk of LVOT obstruction. While there are no set boundaries for neo-LVOT area, a predicted value of $>1.3 \mathrm{~cm}^{2}$ is typically used. The cardiac CT scan is used to determine the access site for the thoracotomy and the location for placement of the sheath in the left ventricle. Patients are placed under general anesthesia for the procedure, with guidance by transesophageal echocardiography (TEE) and fluoroscopy (Figure 2). A small left thoracotomy is performed, followed by placement of left ventricular apical purse string sutures. A 6 or 7 French $(\mathrm{Fr})$ vascular sheath is introduced into the left ventricle over a wire, followed by exchange for the Intrepid ${ }^{\mathrm{TM}}$ delivery catheter. The Intrepid ${ }^{\mathrm{TM}}$ catheter is directed to the left atrium with the assistance of TEE and centered in the mitral valve orifice. The atrial brim is expanded using hydraulic delivery, then aligned on the mitral annulus with care to maintain the brim in the left atrium. The Intrepid ${ }^{\mathrm{TM}}$ valve is deployed during a short run of rapid ventricular pacing. The delivery catheter is then withdrawn from the left ventricle and the apical access site is closed. In general, patients are hospitalized in the intensive care unit for 24 to 48 hours, followed by transfer to telemetry. Patients are placed on warfarin with international normalized ratio (INR) target range of 2.5 to 3.5 for three months, as well as a single antiplatelet agent, consisting of either aspirin $(75,81$, or $100 \mathrm{mg}$ daily) or clopidogrel (75 mg daily).

\section{Patient outcomes}

In 2015, a global feasibility study of the Medtronic Intrepid ${ }^{\mathrm{TM}}$ system was initiated with recruitment from 14 hospitals in Australia, Europe, and the United States (5). For this study, patients with symptomatic, severe MR who were at high or prohibitive surgical risk were considered for enrolment, with primary exclusion criteria being an ejection fraction $<20 \%$, mitral valve calcification, hemodynamic instability, severe pulmonary hypertension, severe renal insufficiency and prior mitral valve surgery or intervention. The results of the initial 50 patients (mean age, $72.6 \pm 9.4$ years; $58 \%$ men; Table 1) who were consecutively enrolled through July 2017 have been published. Severe heart failure was common, with NYHA III or IV symptoms present in $86 \%$. The predominant mechanism of MR was secondary (84\% of patients). The mean left ventricular ejection fraction at baseline was $43.4 \% \pm 11.8 \%$ (range, 20-70\%). Overall, the population was at significantly increased risk of open surgery with an average STS-PROM of $6.4 \% \pm 5.5 \%$ and a EuroSCORE II of $7.9 \% \pm 6.2 \%$. Successful implantation of the Intrepid valve occurred in 48 of 49 attempted patients (98.0\%), with a median procedure time of 100 minutes [interquartile range (IQR), 80, 124] and a median time for device deployment of only 14 minutes (IQR, 12, 17). One malpositioning of the Intrepid valve occurred. There were no incidences of device malfunction or LVOT obstruction and no patient required conversion to open cardiac surgery. Seven deaths occurred within 30 days, with three as a result of apical access site bleeding, three as a result of refractory heart failure and one in the patient with malposition (Table 2).

At a median follow-up of 173 (IQR, 54, 342) days, there were four additional deaths after 30 days (between day 54 and 122) but no deaths after 4 months (Figures 3,4); three of these late deaths were due to sudden cardiac arrest 

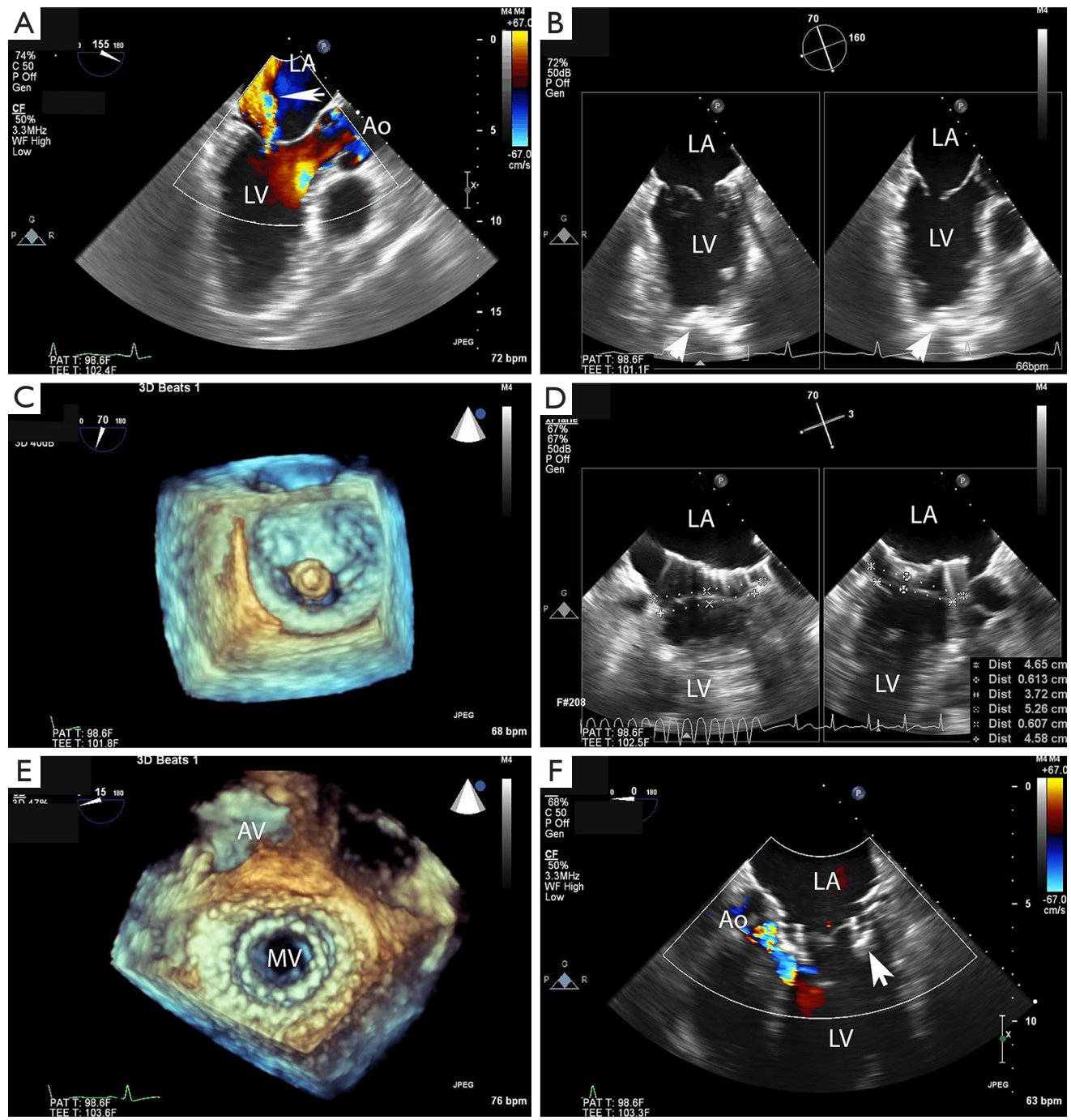

Figure 2 Implantation of the Medtronic Intrepid valve. (A) Baseline transesophageal echocardiography (TEE) demonstrates severe mitral regurgitation (arrow; blood pressure $=128 / 75 \mathrm{mmHg}$ ); (B) following a left thoracotomy, the point of apical access is confirmed manually (arrowheads) with TEE guidance; (C) a 35-Fr sheath is placed transapically and centered in the mitral valve orifice; (D) following deployment of the atrial brim, a 6-mm landing zone is identified on orthogonal views of the mitral valve annulus; (E) three-dimensional TEE (i.e., "surgeon's" view) demonstrates complete deployment; (F) two-dimensional color flow imaging shows no residual mitral regurgitation (blood pressure $=120 / 60 \mathrm{mmHg}$ ). Ao, aorta; AV, aortic valve; LA, left atrium; LV, left ventricle; MV, mitral valve. Reproduced with permission from Bapat et al. (5).

and one due to intracranial haemorrhage in the setting of an unwitnessed fall. Overall, the one-year survival rate was $77 \%$. Mild para-prosthetic MR was present in three patients (7.1\%), while mild prosthetic MR occurred in eight patients (19.0\%) (Figure 5). For the survivors, mild or no symptoms of heart failure (i.e., NYHA functional class I or II) were present in $79 \%(\mathrm{P}<0.0001$ vs. baseline), and, for a subset of patients, there were significant improvements in the Minnesota Living with Heart Failure Questionnaire (MLHFQ) scores $(56.2 \pm 26.8$ vs. 31.7 $\pm 22.1 ; \mathrm{P}=0.011)$ and six-minute walk distance (Figure 6). Pulmonary artery systolic pressure on transthoracic echocardiography decreased from $46.7 \pm 14.7$ to $37.2 \pm 9.7 \mathrm{mmHg}$ at followup $(\mathrm{P}<0.0001)$. No valve degeneration occurred and there were no instances of hemolysis, device embolization or thrombosis. 


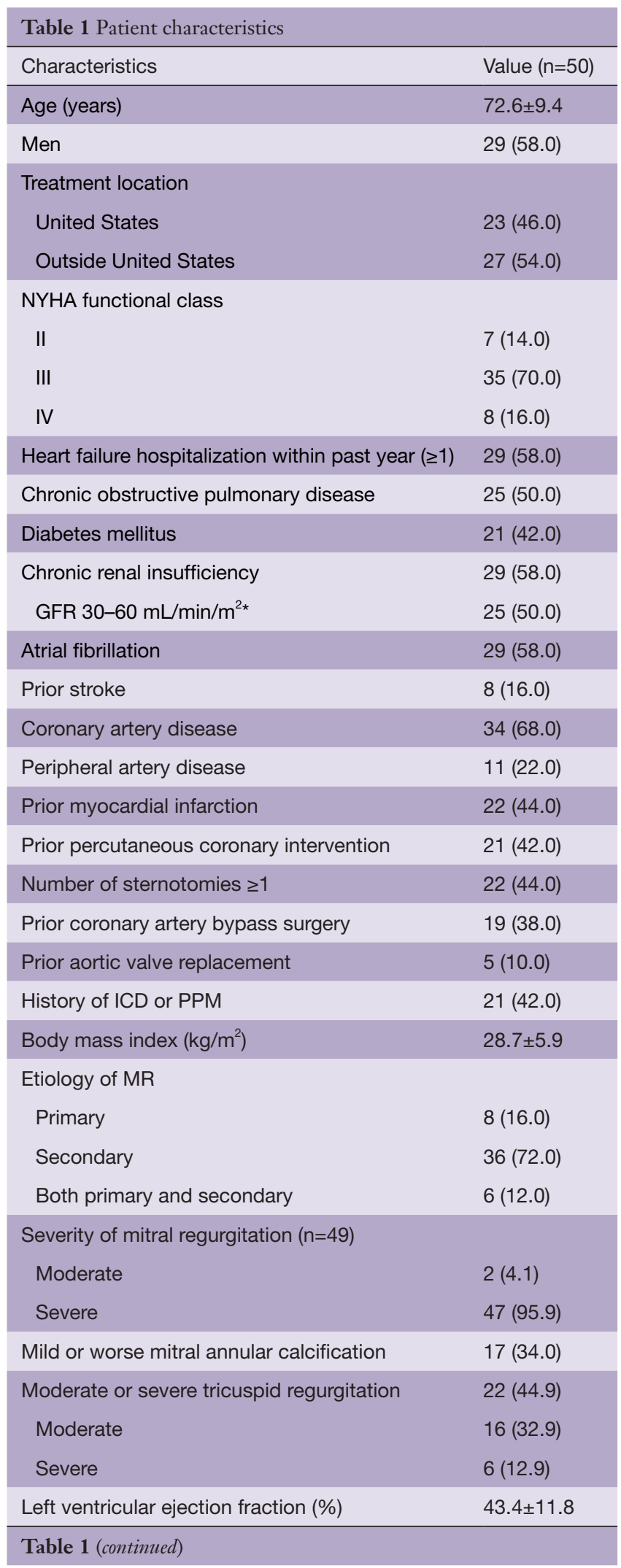

\begin{tabular}{|c|c|}
\hline Characteristics & Value $(n=50)$ \\
\hline \multicolumn{2}{|l|}{ Extenuating circumstances } \\
\hline Frailty & $16(32.0)$ \\
\hline \multicolumn{2}{|c|}{ Pulmonary hypertension (PASP, 55 to $70 \mathrm{mmHg}{ }^{\star *} 20$ (40.0) } \\
\hline Poor mobility & $7(14.0)$ \\
\hline Albumin $<3.3 \mathrm{~g} / \mathrm{dL}^{\#}$ & $9(23.1)$ \\
\hline Anemia $^{\dagger}$ & $22(44.0)$ \\
\hline Liver dysfunction $^{\dagger \dagger}$ & $4(9.5)$ \\
\hline Malignancy & $15(30.0)$ \\
\hline Immunosuppression & $2.0(4.0)$ \\
\hline $\begin{array}{l}\text { STS predicted risk of mortality for mitral } \\
\text { replacement }(\%)\end{array}$ & $6.4 \pm 5.5$ \\
\hline EuroSCORE II (\%) & $7.9 \pm 6.2$ \\
\hline
\end{tabular}

Data are presented as $\mathrm{n}(\%)$ or mean \pm SD. *, patients with GFR $<30 \mathrm{~mL} / \mathrm{min} / \mathrm{m}^{2}$ were excluded; **, patients with PASP >70 mmHg were excluded; ", albumin was not measured in 11 patients; ${ }^{\dagger}$, anemia was defined as haemoglobin $<13.0 \mathrm{~g} / \mathrm{dL}$ in men or $<12.0 \mathrm{~g} / \mathrm{dL}$ in women; ${ }^{\dagger \dagger}$, defined as alanine transferase $>40 \mathrm{U} / \mathrm{L}$ and was measured in 42 patients. GFR, glomerular filtration rate; ICD, implantable cardioverter defibrillator; NYHA, New York Heart Association; PASP, pulmonary artery systolic pressure; PPM, permanent pacemaker; STS, Society of Thoracic Surgeons.

\begin{tabular}{|lll}
\hline Table 2 Adverse events & $\begin{array}{ll}\text { 0-30 days } \\
(\mathrm{n}=50)\end{array}$ & $\begin{array}{l}>30 \text { days } \\
(\mathrm{n}=41)\end{array}$ \\
\hline Events & $7(14.0)$ & $4(9.8)$ \\
\hline Death & $7(14.0)$ & $4(9.8)$ \\
Cardiovascular & $0(0.0)$ & $0(0.0)$ \\
\hline Non-cardiovascular & $2(4.0)$ & $0(0.0)$ \\
\hline Stroke & $0(0.0)$ & $0(0.0)$ \\
\hline Disabling stroke & $2(4.0)$ & $1(2.4)$ \\
\hline Non-disabling stroke & $0(0.0)$ & $0(0.0)$ \\
\hline Myocardial infarction & $5(10.0)$ & $0(0.0)$ \\
\hline Acute renal impairment, stage 3 & $0(0.0)$ & $0(0.0)$ \\
\hline Major vascular complications & $2(4.0)$ & $0(0.0)$ \\
\hline Major cardiac structural complication & $0(0.0)$ \\
\hline Major bleeding & $9(18.0)$ & $1(2.4)^{\dagger}$ \\
\hline Reoperation for any reason & $5(10.0)$ & $0(0.0)$ \\
\hline Reoperation for bleeding & $5(10.0)$ & $1(2.4)^{\dagger}$ \\
\hline Reoperation for other & $0(0.0)$ & $2(4.9)$ \\
\hline New-onset atrial fibrillation & $7(14.0)$ & \\
\hline Table 2 (continued) & & \\
\hline
\end{tabular}




\begin{tabular}{lll} 
Table 2 (continued) & $\begin{array}{l}\text { 0-30 days } \\
(\mathrm{n}=50)\end{array}$ & $\begin{array}{l}>30 \text { days } \\
(\mathrm{n}=41)\end{array}$ \\
\hline Events & $0(0.0)$ & $0(0.0)$ \\
\hline Hemolysis & $1(2.0)$ & $1(2.4)$ \\
\hline Endocarditis ${ }^{\dagger}$ & $0(0.0)$ & $0(0.0)$ \\
\hline Device embolization & $0(0.0)$ & $0(0.0)$ \\
\hline Device thrombosis & $8(16.0)$ & $13(31.7)$ \\
\hline Cardiovascular re-hospitalization & $4(8.0)$ & $8(19.5)$ \\
\hline Re-hospitalization for heart failure & $4(0)$ * reop
\end{tabular}

Data are presented as $n(\%) .{ }^{*}$, reoperation for wound infection at incision site; ${ }^{\dagger}$, the endocarditis event was related to placement of a pacemaker lead.

\section{The APOLLO clinical trial}

A multicenter, pivotal clinical trial was initiated in the fall of 2017 to examine the effectiveness and safety of the Intrepid $^{\mathrm{TM}}$ System in patients with severe, symptomatic MR. The trial consists of two arms, with one-arm randomizing TMVR with $v$ s. traditional surgery $(\mathrm{n}=650)$, and a second arm that will treat patients who are ineligible for surgery as a single cohort $(\mathrm{n}=550)$. For patients in the randomized cohort, the predicted risk of operative mortality is $\geq 3 \%$ with a combined $<35 \%$ risk of mortality or irreversible major morbidity at 30 days, and patients must have an estimated life expectancy of $>24$ months. For the single-arm cohort, the predicted risk of operative mortality

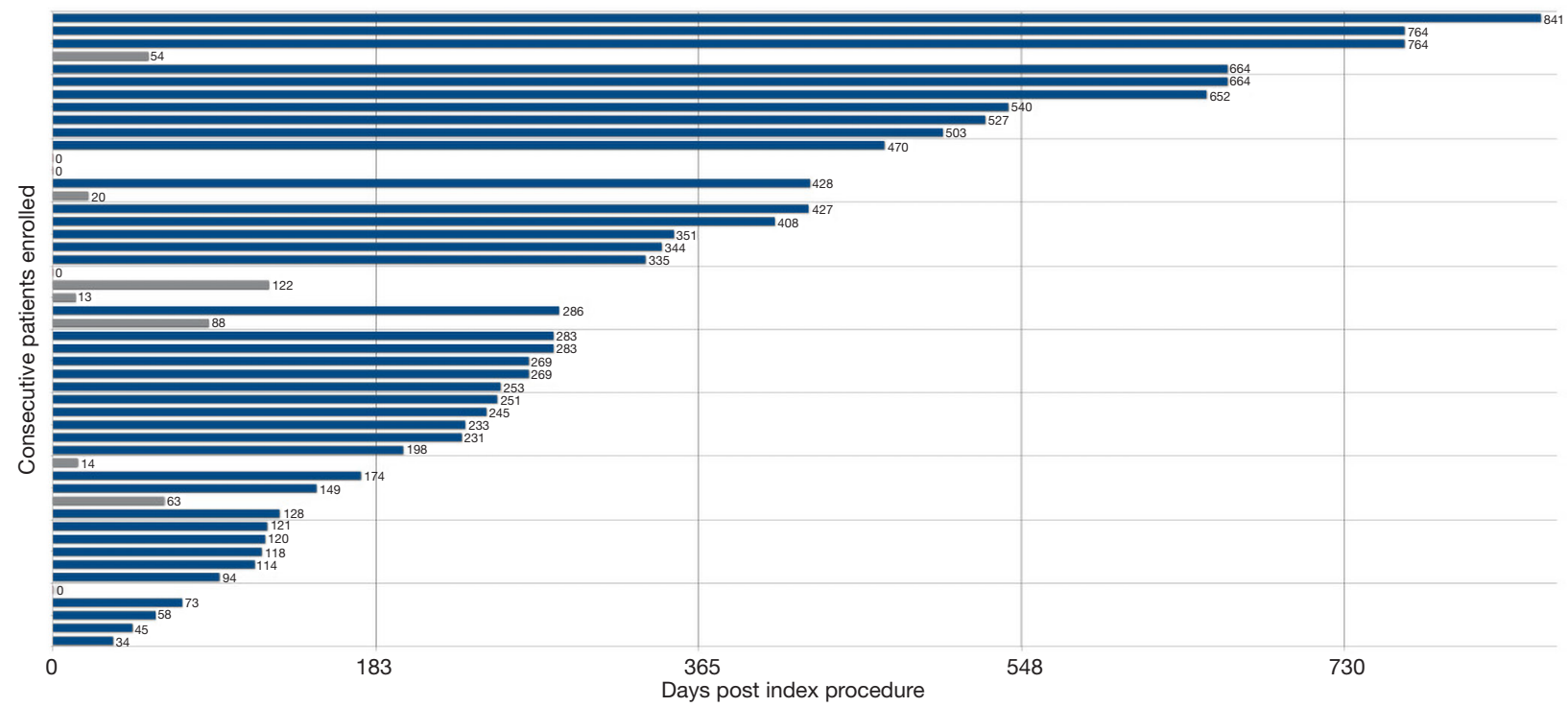

Figure 3 Clinical follow-up for the treated patients. Follow-up time for the 50 patients is illustrated with patients listed on the Y-axis in descending order of treatment. X-axis indicates duration of follow-up in days for each treated patient. All deaths occurred prior to 365 days. Blue = surviving patients; Grey = deceased patients. Reproduced with permission from Bapat et al. (5).
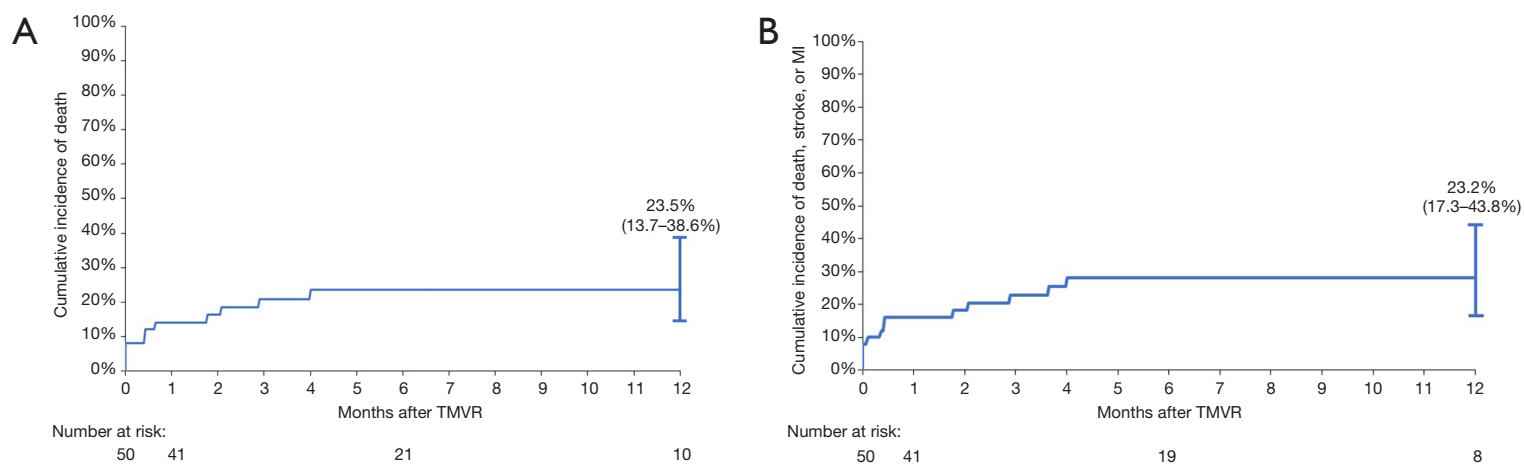

Figure 4 Survival during follow-up. One-year cumulative incidence of death (A) and death, stroke, or myocardial infarction (B) reported as $\%$ (95\% confidence interval). 


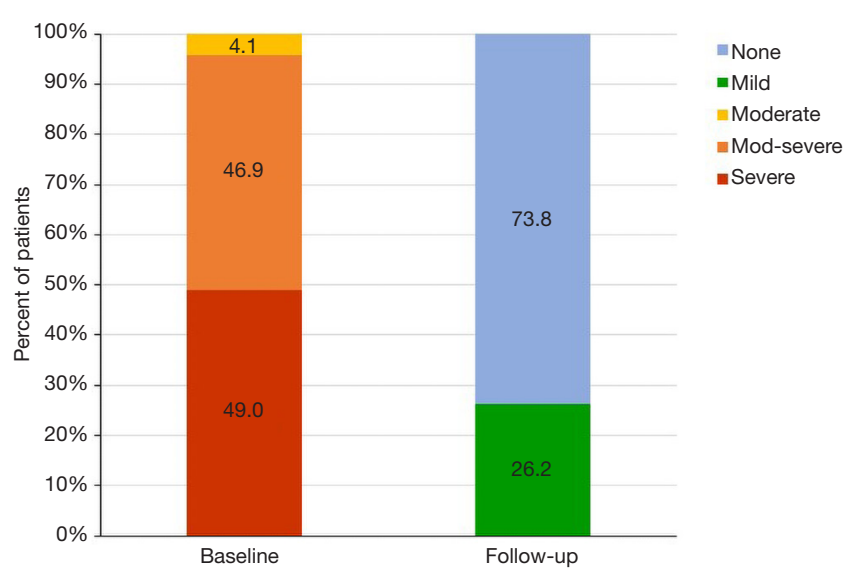

Figure 5 Mitral regurgitation at baseline and follow-up. Reproduced with permission from Bapat et al. (5).

or irreversible major morbidity is $\geq 35 \%$ to $50 \%$ at 30 days and patients must have an estimated life expectancy of $>12$ months. The primary endpoint for the trial is a composite of all-cause mortality, stroke, re-operation (or reintervention) and cardiovascular hospitalization at one year. Secondary safety endpoints include disabling stroke, acute kidney injury, prolonged ventilation, deep wound infection and major bleeding, while efficacy endpoints include the degree of MR improvement, change in quality of life, NYHA class and number of days alive out of hospital at one year.

\section{Conclusions}

Feasibility studies have demonstrated excellent results with the Intrepid system, with over $95 \%$ of patients successfully treated, a minimal learning curve, no device malfunction and improvements in symptom status and quality-of-life among the survivors. For all patients, meticulous attention to apical access techniques and post-operative heart failure care is essential. Ongoing clinical trials will determine the potential role of the Intrepid system compared with surgery and other transcatheter technologies in patients with MR.

\section{Acknowledgements}

None.

\section{Footnote}

Conflicts of Interest: The authors have no conflicts of interest to declare.
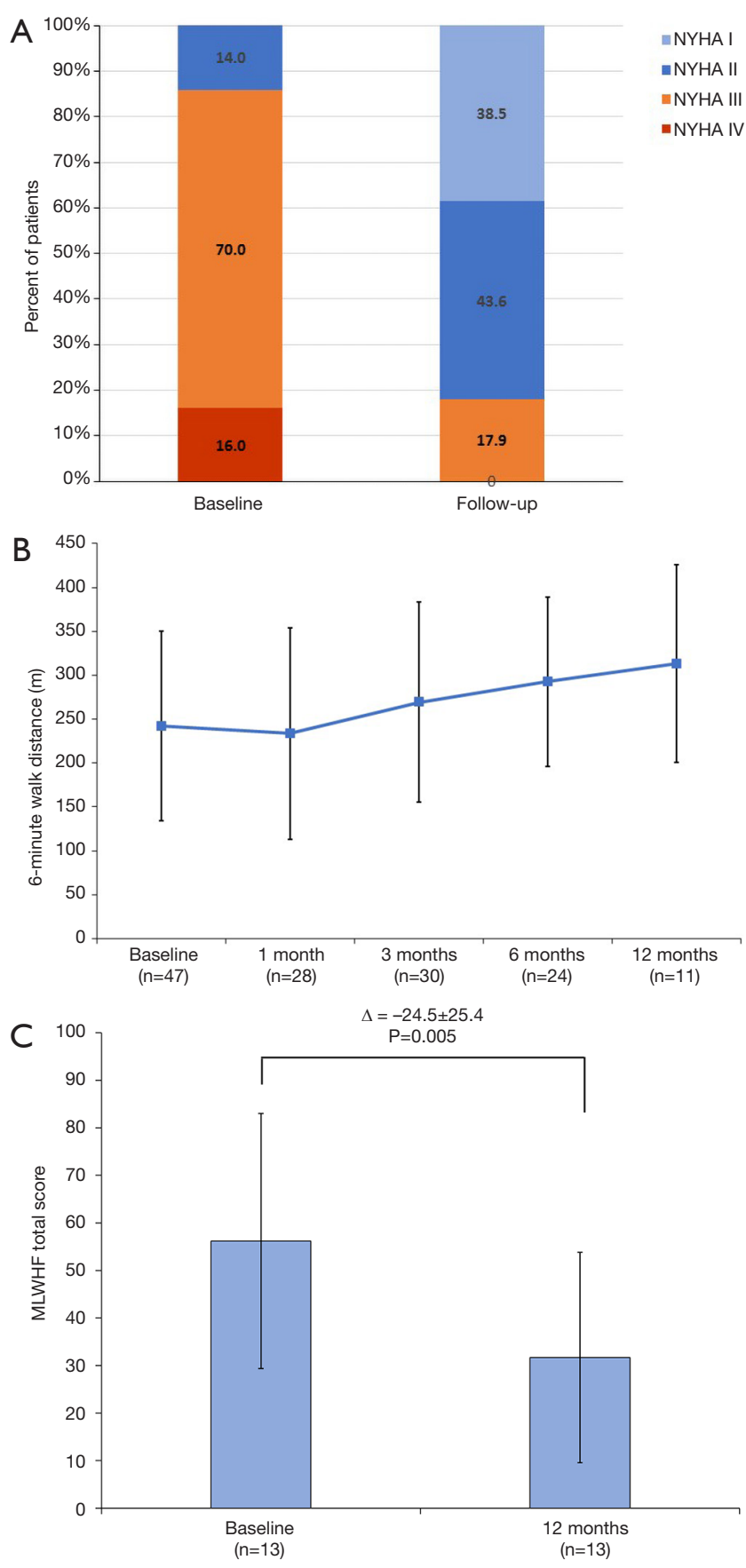

Figure 6 Symptoms and quality of life at baseline and following implantation of the Intrepid valve. (A) Symptom status [New York Heart Association (NYHA) functional class] at baseline, 30 days, and last follow-up; (B) changes in six-minute walk distance; (C) paired data demonstrating change in Minnesota Living with Heart Failure total score. Three patients $(7.1 \%)$ had mild paravalvular MR and eight patients (19.0\%) had mild transvalvular MR graded. Reproduced with permission from Bapat et al. (5). 


\section{References}

1. Nkomo VT, Gardin JM, Skelton TN, et al. Burden of valvular heart diseases: a population-based study. Lancet 2006;368:1005-11.

2. Enriquez-Sarano M, Avierinos JF, Messika-Zeitoun $\mathrm{D}$, et al. Quantitative determinants of the outcome of asymptomatic mitral regurgitation. N Engl J Med 2005;352:875-83.

3. Grigioni F, Detaint D, Avierinos JF, et al. Contribution of ischemic mitral regurgitation to congestive heart failure after myocardial infarction. J Am Coll Cardiol 2005;45:260-7.

4. Meredith I, Bapat V, Morriss J, et al. Intrepid transcatheter mitral valve replacement system: technical and product description. EuroIntervention 2016;12:Y78-80.

5. Bapat V, Rajagopal V, Meduri C, et al. Early Experience With New Transcatheter Mitral Valve Replacement. J Am Coll Cardiol 2018;71:12-21.

6. Blanke P, Dvir D, Cheung A, et al. Mitral Annular Evaluation With CT in the Context of Transcatheter Mitral Valve Replacement. JACC Cardiovasc Imaging 2015;8:612-5.

7. Blanke P, Naoum C, Dvir D, et al. Predicting LVOT Obstruction in Transcatheter Mitral Valve Implantation: Concept of the Neo-LVOT. JACC Cardiovasc Imaging 2017;10:482-5.

Cite this article as: Sorajja $\mathrm{P}$, Bapat V. Early experience with the Intrepid system for transcatheter mitral valve replacement. Ann Cardiothorac Surg 2018;7(6):792-798. doi: 10.21037/ acs.2018.10.03 